\title{
Pourquoi l'acquisition des pronoms est plus simple que celle des articles : apport du japonais L1 dans l'expression de la référence aux entités en français $L 2$
}

\author{
Granget, Cyrille \\ Laboratoire de Linguistique de Nantes (LLING), EA 3827 \\ cyrille.granget@univ-nantes.fr
}

En matière de référence aux entités, les usages malheureux des articles par des apprenants d'une langue première dépourvue d'articles sont souvent envisagés comme le résultat d'un transfert négatif des propriétés morphosyntaxiques de la langue source. Or cette explication qui s'inscrit dans la lignée des hypothèses contrastives présente des limites. Sur le plan théorique, les déterminants du nom ne sont pas des unités absentes du japonais ni d'ailleurs des unités obligatoires en français, et l'explication de leur mésusage dans le français L2 de locuteurs nippophones, par exemple, ne peut résider dans la seule absence de moyens formels en langue première. Sur le plan empirique, cette explication se heurte au constat, non pas d'une absence d'articles dans le discours en langue cible d'apprenants de langue première sans article, mais à un emploi facultatif et une distribution a priori aléatoire des articles (Trévisiol 2003, Sleeman 2004, Lenart 2004, 2012 ). Nous suggérons donc d'envisager également l'hypothèse sémantique selon laquelle les prénoms participent, dans le domaine de la référence aux entités et avec d'autres éléments paranominaux comme les pronoms, de la construction en situation de la structure informationnelle du discours. Nous élargissons ainsi l'étude des articles en français langue seconde à celle des pronoms, autres éléments a priori absents du japonais d'un point de vue strictement morphosyntaxique et présents en français, afin de tester ces deux hypothèses explicatives des usages en situation de contact français L2 - japonais L1. Une revue de la littérature (Lambrecht 1994, Epstein 2002, Lebas-Fraczak 2009) permet de clarifier l'hypothèse sémantique. Elle suggère que, dans une perspective sémantique, les deux notions clés permettant de rendre compte de l'usage des prénoms et pronoms en français sont les notions d'identifiabilité énonciative et de saillance discursive. Si cette dernière notion est pertinente pour décrire l'expression de la référence aux entités en japonais, la précédente l'est beaucoup moins. Ainsi les hypothèses morphosyntaxiques et sémantiques font des prédictions distinctes sur l'usage des prénoms et pronoms en français L2. Tandis que l'hypothèse morphosyntaxique prédit un mésusage des prénoms et pronoms en raison de leur absence et /ou caractère facultatif en langue source, l'hypothèse sémantique prédit que les unités linguistiques qui expriment la saillance en français, en l'occurrence les pronoms, sont, pour des apprenants nippophones, plus accessibles que les unités exprimant l'identifiabilité énonciative, soit les prénoms. Le but de cet article est donc de proposer un cadre translinguistique d'analyse des éléments paranominaux dans les langues en contact et de montrer sa pertinence en deux étapes : d'une part à travers l'étude de la référence aux entités dans des passages narratifs comparables en deux langues, l'une dite sans article, le japonais, et l'autre avec articles, le français. L'analyse contrastive nous montrera la pertinence des notions d'identifiabilité et de saillance pour comprendre l'expression de la référence aux entités dans ces deux langues. D'autre part, une étude longitudinale exploratoire de l'usage des prénoms et pronoms dans le discours narratif en français L2 d'apprenants nippophones à partir du même support en image, nous montrera que le processus acquisitionnel en situation de contact japonais L1-français L2 est prioritairement guidé, non pas par des contraintes morphosyntaxiques, mais par des catégories sémantiques.

\section{Etat des lieux}

On peut distinguer dans la littérature acquisitionnelle consacrée aux usages malheureux des articles définis et indéfinis en langue seconde deux façons d'envisager ce phénomène. La première considère que les articles sont des moyens grammaticaux présents dans certaines langues dites langues avec articles et 
absentes d'autres langues dites langues sans article. Dans cette perspective, la tâche de l'apprenant est d'acquérir une catégorie grammaticale signifiante: le principe d'antéposition quasi obligatoire de l'article devant le nom et les distinctions sémantiques respectivement encodées par les articles défini et indéfini. Les notions de transfert positif ou négatif (Zobl 1984) ou l'absence en langue source de catégorie grammaticale encodant morphosyntaxiquement la définitude (Sleeman 2004) sont les principales raisons invoquées pour expliquer les difficultés acquisitionnelles. Même si l'absence d'article correspond aussi à un stade de développement (Schumann 1978), Zobl (1984) relève des facilités acquisitionnelles selon que les langues premières des locuteurs possèdent la catégorie grammaticale des articles, lesquelles se traduisent par des différences de rythme d'acquisition: l'acquisition de $a$ et the en anglais L2 est plus rapide pour les locuteurs dont la langue première fait la distinction entre des déterminants définis et indéfinis (français, espagnol) que pour ceux qui ne la font pas (chinois, russe). Sleeman (2004) montre qu' à compétence narrative égale, les étudiants de L1 japonais (LsA) font plus d'erreurs à l'oral que les néerlandophones $(\mathrm{LaA})$. L'auteur relève des phénomènes tels que (i) l'absence d'articles devant le nom, dans 6,6\% des références aux entités dans la narration orale de Frog Story (33/502, 9 locuteurs, ex. : grenouille est sortie dans le pot, ils cherchaient grenouille Sleeman $2004: 7$ ), (ii) l'emploi d'un article indéfini devant un nom référant à une entité déjà introduite (aucun cas chez les néerlandophones), ex : le garçon et le chien a trouvé la grenouille n'est pas dans le pot, un garçon a ouvert la fenêtre, ou encore (iii) l'emploi a contrario d'un article défini devant un nom référant à une entité nouvellement introduite, il $y$ avait un garçon et un chien, ils avaient une grenouille dans le pot. Cette perspective soulève au moins trois questions. L'idée que les articles définis et indéfinis expriment les référents anciens et nouveaux rend-elle compte du fonctionnement discursif réel de ces éléments ? Sachant qu'il existe des déterminants du nom et compléments du nom pré-nominaux en japonais, le concept de prénominalisation est-il complètement étranger aux apprenants nippophones ? Les cas d'omission ou de distribution non cible des articles sont-ils imputables à l'absence de cette catégorie grammaticale en langue source ou, plus globalement, à l'absence de moyens linguistiques équivalents du point de vue du sens ?

Une deuxième façon d'envisager l'acquisition des articles consiste à considérer que ceux-ci participent, avec d'autres moyens paranominaux comme les pronoms, de la référence aux entités et de la construction de la structure informationnelle du discours. Dans cette perspective, la tâche de l'apprenant est d'acquérir en L2 les moyens formels d'encoder des notions sémantiques potentiellement encodées de manière grammaticale, lexicale ou discursive en L1. Cette conception s'appuie donc sur le double postulat d'interopérabilité entre moyens grammaticaux, lexicaux et discursifs et de primauté des catégories sémantiques dans le processus psycholinguistique d'acquisition d'une langue seconde. Dans ce paradigme de recherche, on peut noter des résultats et des questions. Les études menées (Hendricks 2000, Jarvis 2002, Trévisiol 2003, Lenart et Perdue 2004, Lenart 2012) montrent que les articles participent avec l'ensemble des déterminants et les pronoms à la construction d'une structure informationnelle cohérente. Lenart (2012) remarque ainsi que dans les récits d'apprenants polonophones débutants, les syntagmes nominaux dépourvus d'articles contiennent souvent d'autres constituants paranominaux, complément du nom postposé ou adjectif antéposé (ex : ami de Reksio, petit chien,). Lenart montre aussi l'émergence en L2 de moyens intermédiaires comme la proto-construction présentative : il s'agit de, c'est une histoire de chien, ce film raconte, etc. Ces constructions présentent des traits caractéristiques des constructions présentatives relatives (CPR) telles que il y a un garçon qui ... mises en avant par Lambrecht (2000) dans sa description du français mais ne remplissent pas tous les critères, comme l'emploi d'un pronom relatif. L'émergence de la proto-construction présentative et de moyens de complémenter le nom montre que l'absence d'article est compatible avec une acquisition en cours des moyens linguistiques de structurer l'information et d'exprimer la référence aux entités dans le récit. Elle suggère que l'absence d'articles pourrait être compatible avec l'expression sous une autre forme linguistique des principes qui régissent leur emploi et leur distribution.

La nature des principes qui régissent l'emploi des articles définis et indéfinis est une question essentielle qui nécessite des clarifications. Les catégories informationnelles souvent avancées d'introduction et de maintien de la référence à une entité ne rendent compte en effet que d'une partie des usages des articles définis et indéfinis. Jarvis (2002) conclut ainsi que cette distinction informationnelle entre l'information topique, ou donnée, et l'information commentaire, ou nouvelle, est problématique, "not least because of 
the different views on how to define this distinction ». S'interrogeant sur l'universalité de cette notion centrale souvent appelée topique, l'auteur en appelle à des travaux pour définir si cette notion est véritablement opératoire pour rendre compte des développements linguistiques en situation de contact de langues. C'est pourquoi, avant de procéder à l'anlyse des données, nous nous emploierons à clarifier les notions informationnelles exprimées par les articles définis et indéfinis, ainsi que par les pronoms en français.

Nous élargissons l'étude de l'expression de la référence aux entités aux pronoms afin de comprendre quelle hypothèse guide les apprenants dans l'acquisition des moyens linguistiques de référer aux entités au moyen d'un deuxième observatoire. En effet, comme les articles, les pronoms sont des catégories morphosyntaxiques absentes de la langue source. Comme à la question de savoir si en situation de contact de langue, l'absence d'une catégorie morphosyntaxique dans une langue source prédit des usages inappropriés dans une langue cible, l'hypothèse morphosyntaxique répond par l'affirmative, elle prédit donc dans la situation observée un usage inapproprié tant des prénoms (articles) que des pronoms, en raison même de leur absence en langue source. L'hypothèse sémantique prédit en revanche que l'usage d'un moyen linguistique en langue cible dépend de l'expression en langue source de la notions sémantique afférente. En d'autres termes, si l'usage des prénoms et pronoms en français relèvent d'une catégorie sémantique exprimée en langue première, alors ces unités sont apprenables.

Avant de poursuivre nos investigations et de clarifier les prédictions de l'hypothèse sémantique au sujet de l'apprenabilité des prénoms et pronoms, il convient donc de préciser, de façon théorique dans un premier temps, de quelles catégories sémantiques relèvent ces unités linguistiques.

\section{De quoi les prénoms et les pronoms sont-ils le nom ?}

C'est volontairement que nous n'emploierons pas la notion de topique souvent employée dans les études sur l'acquisition de la référence aux entités. Sachant qu'elle est utilisée pour référer à des phénomènes distincts, il nous semble préférable d'employer dans ce cas des termes distincts et de réserver les notions de topique et de commentaire à leur acception informationnelle d'information donnée versus nouvelle dans le cadre strictement discursif. Nous nous attacherons ainsi aux notions d'identifiabilité énonciative qui explique l'usage des articles et souligne le lien entre la structure informationnelle et le cadre de l'énonciation, et de saillance cognitive qui explique les procédés de hiérarchisation de l'information dans le discours et notamment l'emploi des pronoms.

\subsection{La notion énonciative d'identifiabilité}

Comme l'a déjà montré Epstein (2002) à propos de l'anglais, il est abusif de considérer que les articles défini et indéfini encodent les notions informationnelles de donné/nouveau, comme le montre l'échange, heureux, suivant :

(1) Pierre arrive en salle de réunion à l'heure prévue, un seul collègue est présent, il lui demande :

1a - Où sont les collègues?

La personne répond

$1 \mathrm{~b}$ - ben Martine est partie à la piscine et Léo et Mathieu sont partis voir le film qui a été primé à Cannes là... j'ai oublié le titre

Dans cet échange imaginaire mais plausible sur le plan linguistique, bien que les entités $<$ collègues $>$, $<$ piscine $><$ film $>$ et $<$ titre $>$ désignées soient nouvelles en discours, elles sont encodées au moyen d'un nom précédé d'un article défini. L'article défini ne peut donc être considéré comme un moyen d'exprimer strictement l'information ancienne. En 1b, piscine renvoie à un lieu qui fait partie d'un univers spatial de référence partagé par le locuteur et l'interlocuteur. L'emploi de cet énoncé suppose que les entités « piscine » ou «film» font partie du lieu élargi où a lieu l'énonciation ou d'un univers de référence commun à l'interlocuteur et au destinataire. Cet énoncé n'est pas recevable ou suscitera des interrogations si l'énonciateur se trouve dans une station d'observation au pôle nord. Il ne s'agit pas de rejeter 
complètement le pouvoir explicatif des notions informationnelles d'information ancienne et nouvelle mais de les considérer dans leur contribution à la définition d'une notion dont le pouvoir explicatif est plus grand, la notion d'identifiabilité énonciative. On peut en effet considérer avec d'autres (Epstein 2002, Lebas-Fraczak 2009) que les articles expriment l'identifiabilité d'un référent en situation d'énonciation. Au moyen de l'article défini, le locuteur signale à l'allocutaire l'identifiabilité de la référence de l'entité mentionnée; au moyen de l'indéfini, il signale que la référence n'est pas identifiable. Plusieurs raisons permettent de supposer qu'un référent est identifiable :

- Il a déjà été mentionné en discours, il a donc un statut informationnel « donné » versus «nouveau ». Autrement dit, c'est, dans le récit, un « topique », au sens restreint.

- Une entité avec laquelle il entretient un lien a déjà été nommée, c'est le cas de l'anaphore associative (ex, le titre en $1 \mathrm{~b})$.

- Il est matériellement présent dans la situation énonciative ou inférable de celle-ci (ex les collègues en 1b)

Ainsi une entité peut avoir un statut informationnel «nouveau » en discours et être encodée au moyen d'un article défini du fait qu'elle constitue un élément immédiatement identifiable dans la situation d'énonciation. Les notions strictement discursives de topique/commentaire sont nécessaires mais pas suffisantes pour expliquer l'ensemble des emplois des articles. Cette clarification terminologique nous semble indispensable pour aborder la complexité de l'acquisition des articles par des locuteurs d'une langue dite sans article.

\subsection{La notion discursive de saillance}

La notion énonciative d'identifiabilité est en effet à distinguer de la notion discursive de saillance qui explique, entre autres, l'usage préférentiel des pronoms plutôt que des noms en situation discursive de maintien de la référence. Ici aussi, cette notion de saillance discursive ne doit pas être confondue avec celle d'information donnée ou de topique, elle correspond au «sujet dont il est question » ou à la notion dite en anglais d'aboutness. Plusieurs auteurs (Givon 1983, Lambrecht 1994) ont proposé une échelle de saillance (appelée différemment selon les auteurs) qui permet d'expliquer la propension des locuteurs à employer des noms ou des pronoms pour référer à des entités en discours. Lambrecht (2000) considère également que le français dispose d'une structure, la construction présentative relative (CPR), qui permet d'exprimer le statut saillant que le locuteur assigne à une entité nouvellement introduite en discours. Cette construction est indépendante de l'article, défini ou indéfini, employé dans le SN qui réfère à l'entité. Dans l'énoncé c'est l'histoire d'un garçon qui joue avec son chat, l'emploi de la construction présentative relative est indépendante des articles. La CRP s'explique par la construction discursive en début de récit d'une unité saillante, tandis que les articles rendent compte de l'identifiabilité des référents dans la situation d'énonciation. Notons que la saillance s'exprime prioritairement en français par la CRP et par la fonction syntaxique sujet du nom ou du pronom. Comme le note déjà Chafe (1976), les SN sujet sont plus saillants que les SN objet. La saillance est donc une notion discursive, syntaxique et également subjective car il appartient à chaque sujet locuteur de faire en discours un choix de conceptualisation qui rend telle entité plus saillante que telle autre. En général, la réalité extralinguistique détermine des possibilités mais le locuteur a aussi le choix de promouvoir dans le discours une entité au statut d'entité principale ou discursivement saillante à propos de laquelle il y a quelque chose à dire. La saillance s'exprime donc par des moyens discursifs (CRP) mais aussi morphosyntaxiques (sujet, pronom).

Nous avons précisé de façon théorique de quelle catégories sémantiques relèvent les articles définis et indéfinis et les pronoms en français. Afin de faire parler l'hypothèse sémantique, il importe maintenant de répondre à la question de savoir si ces notions centrales d'identifiabilité énonciative et de saillance discursive sont exprimées en japonais. 


\section{Identifiabilité et saillance discursive en japonais}

La notion énonciative d'identifiabilité exprimée en français de façon quasi systématique dans le syntagme nominal par les articles définis et indéfinis n'a pas d'équivalent en japonais sur un plan formel et fonctionnel. Il n'existe pas en japonais d'éléments morphosyntaxiques prénominaux employés de façon systématique pour exprimer l'identifiabilité des référents dans la situation d'énonciation. Hidetake (2010) souligne que « le trait [+/- défini] est toujours marqué morphologiquement par le déterminant en français alors qu'en japonais les marqueurs de ce trait sont facultatifs. » Il existe certes en japonais quelques déterminants prénominaux: kono, sono, ano (démonstratif) pour le défini et aru (certain), hitotsu no (un, numéral cardinal), nanika (quelque chose), etc. pour les indéfinis. L'auteur explique toutefois que l'identifiabilité de la référence, qu'il exprime en terme de préconstruction de la référence, est souvent interprétable de façon univoque en situation d'énonciation. Il illustre son propos au moyen de deux énoncés distincts par la seul trait [+/- défini] et qui reçoivent une expression identique en japonais :

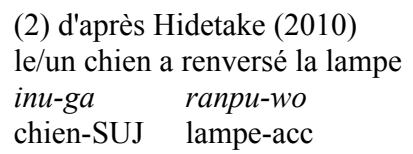

En effet, dans l'énoncé (2), soit les référents de inu/chien et lampu/lampe se trouvent dans un domaine préalablement construit par le contexte immédiat, la connaissance de la situation d'énonciation, la connaissance encyclopédique du monde, etc. et il est interprété comme identifiable, soit il se trouve dans aucun domaine préalablement construit, il est nouvellement introduit dans la situation d'énonciation et est interprété comme non identifiable. Dans le cas de l'énoncé ci-dessus, si les locuteurs partagent la connaissance qu'il y a un chien et une lampe spécifiques dans la situation d'énonciation, l'interlocuteur identifie ces objets spécifiques comme les référents visés par les noms. L'identifiabilité en japonais étant inférable de la situation d'énonciation globale, il n'est pas nécessaire de la marquer. Sous cet angle, le marquage morphologique du français apparaît comme une opération linguistique redondante.

Il existe selon Hidetake quelques cas où l'identifiabilité du référent doit être marquée car l'interprétation visée diffère de l'interprétation par défaut inférable de la situation d'énonciation. Dans ce cas, l'emploi d'un déterminant indéfini comme aru permet d'expliciter le trait « non identifiable ».

La saillance discursive d'une entité est en revanche une notion centrale en japonais exprimée au moyen d'un syntagme nominal qui y réfère en position initiale d'énoncé, suivi d'une particule - wa. «avec WA », écrit Klingler (2003:169), «le N est saisi comme renvoyant à un référent accessible dans le champ discursif des deux interlocuteurs ». La construction de l'énoncé en WA est un mode de liaison corrélative ou d'insertion des groupes nominaux dans l'énoncé (Tamba et Terada 1991) qui se distingue d'un autre mode d'insertion, au moyen de la particule -GA postposée au nom. L'énoncé N + GA ..... SV est un énoncé monolithique : le Nom est dans la dépendance du Verbe. Le locuteur fait prioritairement référence à un événement qui implique des entités. La particule dite casuelle marque le statut syntaxique du SN par rapport au verbe, elle ne marque pas une hiérarchie dans la structure informationnelle. En revanche, l'énoncé N + WA .......SV est un énoncé bipartite : le Nom est détaché de l'énoncé au moyen de WA. Le locuteur fait prioritairement référence à une entité au sujet de laquelle est faite une assertion. En ce sens, on peut considérer que l'entité est discursivement saillante. Tamba et Terada (1991) notent d'ailleurs que «WA instaure une rapport de corrélation ordonné entre deux parties de l'énoncé que l'on peut rapprocher des parties nommées dans la linguistique occidentale topique/commentaire » Les auteurs précisent toutefois qu'ils entendent la notion de topique au sens d'entité discursivement saillante, «topique étant ici à entendre comme le sujet dont il est question et non dans son sens informationnel d'information donnée ». La particule WA en japonais constitue donc un moyen linguistique de hisser une information au statut d'information saillante dans le discours.

Si l'on admet que l'identifiabilité des référents ne donne pas lieu à un marquage linguistique explicite et systématique en japonais mais que la saillance discursive est une notion pertinente pour décrire la structure informationnelle et la référence aux entités en japonais, alors on peut postuler que, si l'hypothèse sémantique est valable, alors les apprenants nippophones du français langue seconde 
devraient dans les premiers stades d'acquisition rencontrer des difficultés dans l'usage des articles en français en raison de l'inaccessibilité du principe d'identifiabilité qui régit leur usage mais des difficultés moindres, voire nulles, dans l'usage des pronoms en raison de l'accès, ouvert en langue source, à la notion de saillance discursive. Nous allons donc pouvoir confronter cette hypothèse sémantique à l'hypothèse morphosyntaxique selon laquelle l'acquisition des prénoms et des pronoms est un processus d'un degré de difficulté semblable en raison de l'absence de ces catégories grammaticales obligatoires en japonais, langue source.

\section{Dispositif de recueil des données}

Afin de tester ces deux hypothèses, nous disposons d'un corpus de données narratives fictionnelles recueillies auprès d'apprenants nippophones du français langue seconde, puis complétées par des données en français et japonais langues premières ${ }^{1}$.

\subsection{Support}

Le support utilisé pour recueillir l'ensemble des récits fictionnels en français L2, français L1 et japonais L1 est le même : il s'agit d'une bande dessinée de dix vignettes en couleurs de format A4 et intitulée L'histoire du chat. Le scénario mis en images par Miki Minato n'est pas sans rappeler Frog Story (Meyer 1969) : lors d'un moment d'inadvertance, un chat échappe à l'attention de son maître, un jeune garçon, qui part alors à sa recherche, questionne à ce sujet les différentes personnes qu'il rencontre et finit par le retrouver. Cette histoire simple présente un protagoniste principal qui apparaît dans toutes les vignettes, et des protagonistes secondaires, parmi lesquels un protagoniste qui apparaît dans les deux premières et deux dernières vignettes mais reste présent en toile de fond puisqu'il fait l'objet de la quête du personnage principal : le chat. Le récit de cette histoire en images est donc susceptible de donner lieu à l'expression de continuités et de ruptures référentielles selon qu'il est fait référence à un protagoniste ou à un autre.

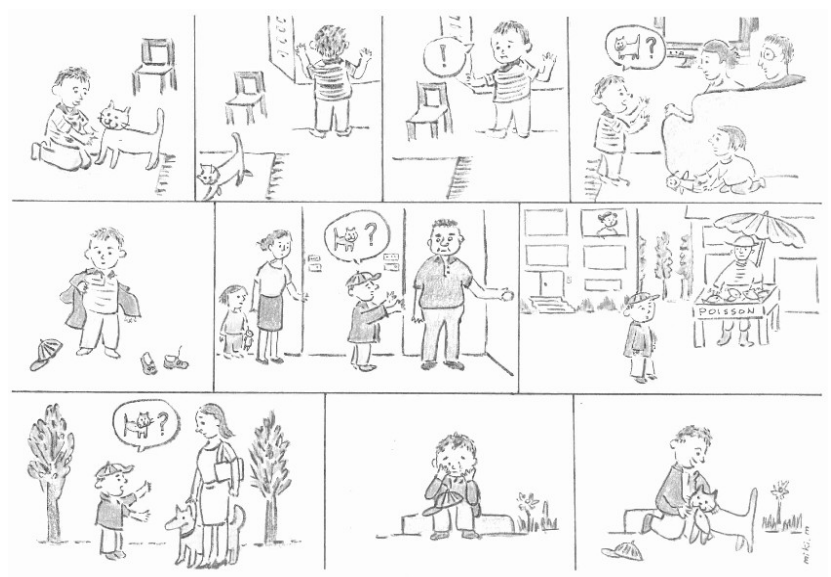

Histoire du chat, Miki 2007(c)

\subsection{Participants}

Les récits se répartissent en trois groupes selon la langue et son statut pour le locuteur. Le premier groupe, « français L2 », est constitué de 9 étudiantes inscrites en 2ème et 3ème année d'un cursus de « Langue et littérature françaises » dans une université japonaise. Elles ont raconté l'histoire à deux reprises : une première fois, à un moment dit $\mathrm{t} 1$, au début du mois de juillet au Japon et une deuxième fois deux mois plus tard, à un moment dit $\mathrm{t} 2$, en France, à la fin d'un séjour d'immersion. Le deuxième groupe « français L1» est constitué de six étudiants dans la même fourchette d'âge, vivant en France au moment de l'enregistrement et dont le français est la langue première. Ils ont raconté l'histoire en français. Le 
troisième groupe «Japonais L1 » est constitué d'étudiants vivant en France et dont le japonais est la langue première. Ils ont raconté l'histoire en japonais.

\subsection{Recueil et analyse des données}

Les récits ont été recueillis en situation de face à face par deux enquêteurs. Le narrateur en français L2 devait parmi plusieurs enveloppes en une qui contenait une planche de vignettes. Ce faux-choix (toutes les enveloppes contenaient la même planche) visait, avec une attitude distanciée de l'enquêteur, à simuler un déséquilibre informationnel et à stimuler l'échange d'informations. La consigne de l'activité proposée a été donnée en français aux locuteurs des trois groupes : racontez-moi l'histoire s'il vous plait. Ce message a été assorti d'un nihon-go de oshiete kudasaï (racontez moi en japonais) pour les locuteurs nippophones $\mathrm{du}$ japonais L1. Les récits ont été enregistrés et transcrits sous forme principalement orthographique au moyen du logiciel EXMARaLDA, glosés et traduits en français pour les récits en japonais.

Dans la présente étude, l'analyse porte sur les débuts de récits correspondant à la verbalisation des trois premières vignettes. Celles-ci impliquent deux protagonistes dans trois scènes différentes : a. le garçon et le chat jouent ensemble, b. le garçon va à la fenêtre et le chat s'enfuit, c. le garçon se retourne et le chat n'est plus là. Notre méthode d'analyse a consisté à repérer les différents moyens linguistiques (pronoms, construction relative présentative, prénoms - articles définis, indéfinis, posessifs) de référer aux entités $<$ garçon $>$ et $<$ chat $>$ (i) dans l'énoncé, (ii) d'un énoncé à l'autre, (i) d'un récit à l'autre, et de comprendre ce qui détermine la variation des expressions de la référence aux entités dans les trois langues observées, français L1, japonais L1 et japonais L2. La restriction de l'analyse aux débuts de récit vise, dans cette étude exploratoire, à définir de manière qualitative un cadre d'analyse généralisable à une étude de plus large portée.

\section{Résultats}

\subsection{L'expression de la saillance en français et en japonais}

\subsubsection{En français $L 1$}

La nécessaire linéarisation de la parole oblige à ordonner la référence aux entités. Dans les récits recueillis, la première entité à laquelle le narrateur fait référence est <garçon>. Elle est exprimée au moyen d'un syntagme nominal un ptit garçon qui est le sujet de l'énoncé. Dans cinq récits sur six, les locuteurs emploient aussi une construction relative présentative (CRP) introduite par un verbe de présentation comme en (3) ou de perception comme en (4) :

\section{(3) L5}

donc il y a un ptit garçon qui joue avec son cha:

(4) L1

hm dans la première image on peut voir un ptit garçon qui joue avec son chat

La construction relative présentative promeut une entité au rang d'entité saillante, une fonction redondante par rapport à la fonction syntaxique sujet et à la fonction sémantique d'agent du syntagme nominal.

Le caractère saillant de cette entité ainsi promue implique que, si l'énoncé (5) suivant commence par un pronom personnel sujet masculin, il ressort qu'en raison de la compétition référentielle entre les coréférents $<$ garçon $>$ et $<$ chat $>$, c'est $<$ garçon $>$ qui coréfère avec le pronom.

(5)

donc il y a un ptit garçon qui joue avec son chat: IL est sale 
Le pronom $i l$ en (5) coréfère non pas avec le nom chat qui le précède mais avec petit garçon. En ce sens, on peut considérer que < garçon> est une entité saillante dans le récit à ce moment de l'énonciation et que, d'une manière générale, l'entité en fonction sujet est plus saillante que les autres entité. Le pronom n'est toutefois qu'un révélateur de saillance, il ne la construit nullement, comme nous pouvons également l'observer dans l'énoncé (6) suivant:

\section{(6) L3}

aloreuh c'est un ptit garçon qui joue avec son cha dans sa maison

euh a un moment donqu' i se:: retourn' euh::

et euh i tourne lae dos a son chat en fait qui euh qui s'en va

et quand i's'retourn' i's'rend compte en fait que son chat n'est plus la

donqu' il'est assez perdu

Si le pronom personnel sujet maintient ainsi la référence à l'entité saillante <garçon>, mentionnée en position de sujet dans l'énoncé précédent, un syntagme nominal sujet exprime en revanche un changement de sujet syntaxique et donc un changement d'unité saillante, comme en (7) ci-dessous :

\section{(7) L5}

donc il y a un ptit garçon qui joue avec son cha:

euh ils sont tous les deux

a un moment le ptit garçon regard'par la f'nêtre

et le chat s'en va

et quand leptit garçon se r'tourne

il a disparu 'fin le chat a disparu

et donc le ptit garçon se pose des questions

Dans ce début de récit, l'alternance régulière de la référence à $<$ garçon $>$ et $<$ chat $>$ d'une proposition à l'autre s'exprime par l'emploi d'un nom. L'emploi par le locuteur d'un pronom pour référer à $<$ chat $>$ mentionné par un nom dans l'antépénultième énoncé, est suivi quasi immédiatement d'un emploi autocorrectif d'un nom pour désambiguïser la référence. En effet, le ptit garçon est sujet de l'énoncé précédent et constitue donc l'entité saillante avec laquelle coréfère immédiatement le pronom $i l \mathrm{du}$ fait de propriétés de genre identiques. Or à ce moment de l'énonciation, le locuteur fait référence à lentité $<$ chat $>$. Ce même phénomène auto-correctif s'observe dans l'extrait (8) suivant dans lequel le narrateur emploie dans un premier temps, pour référer à $<$ garçon $>$, un pronom qui, dans le cotexte du récit, réfère à l'entité <chat>, puis s'autocorrige en utilisant un syntagme nominal qui instaure clairement dans le récit une nouvelle entité saillante à laquelle se rapporte l'assertion.

\section{(8) L6}

donc sur la première image ya un pti garçon qui:: qui yest avec son chat dans le dans un salon et:: sur la deuxième apparemment $i$ joue à cache cache et l'cha fait semblant d'aller s'cacher sur la troisèmle il se ret 'fin il lepti garçon ser'tourne et donc i voit plus le ptit chat

L'emploi du pronom personnel sujet en cotexte approprié constitue donc un bon indice de l'expression de la notion de saillance. Il exprime le fait que les unités dans la phrase n'ont pas la même accessibilité selon qu'elles sont en position sujet ou en position objet. Si dans un énoncé le nom animé sujet et un autre nom animé sont de même genre, un pronom personnel sujet de même genre dans l'énoncé suivant ne coréfère pas avec le dernier nom mentionné, mais avec le nom sujet de l'énoncé précédent.

Si l'on quantifie ces phénomènes, on peut noter, parmi les 6 débuts de récits ( 3 vignettes) analysés, quelques tendances fortes dans l'expression de la référence aux entités dans les récits en français L1:

- Dans tous les récits, petit garçon est sujet du premier énoncé.

- Dans 5 récits sur 6, le sujet est enchâssé dans une construction relative présentative (CRP).

- Parmi les 15 cas de référence en fonction de sujet à une entité exprimée dans l'énoncé précédent par un SN sujet, le sujet est un pronom personnel dans les 15 cas recensés. 
- Parmi les 14 cas de référence, en fonction sujet, à une entité exprimée dans l'énoncé précédent par un SN non sujet, le sujet est un syntagme nominal dans 12 cas et un pronom, personnel (1) et relatif (1), dans les deux autres cas.

L'analyse des débuts de récits en français montre donc la pertinence de la notion translinguistique de saillance discursive et de l'usage des pronoms personnels sujets en cotexte our rendre compte de l'expression linguistique de la saillance.

\subsubsection{En japonais}

La linéarisation permet aussi d'observer la hiérachie des syntagmes nominaux en japonais. Dans les débuts de récit en japonais, le nom pour <garçon $>$ est systématiquement dans un syntagme nominal en position initiale du premier énoncé du récit. Nous observons dans les deux premiers énoncés l'usage systématique de la particule wa, qui détache une unité de l'énoncé à propos de laquelle vaut l'assertion. Ce détachement est exprimé dans le premier énoncé comme en (9) ou dans le deuxième comme en (10b), auquel cas le premier énoncé du récit expose le ou les protagoniste(s) :

(9) Misa

tanoku wa tanoku no neko taihen kawaï datte i-mash-ta gars wa gars rel chat très mignon être-Pass Cop-Pass le gars, son chat était très mignon

(10) Kaho

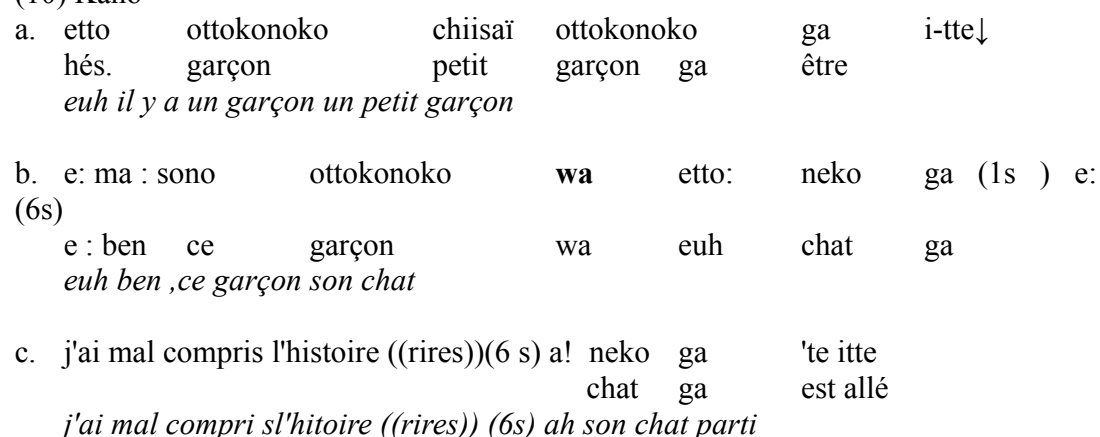

Dans l'ensemble des récits, <garçon> est donc aussi la première entité promue au statut d'entité saillante au moyen de $w a$.

La continuité référentielle s'exprime par une série de verbes référant à des situations impliquant l'entité ou une autre, dans la portée du syntagme nominal suivi de wa:

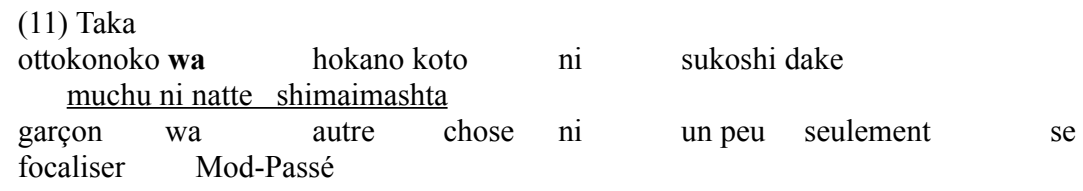

le garçon malheureusement détourne un court instant son attention vers autre chose

sono skoshi dake $\begin{aligned} & \text { mutchu ni natte i-tta } \\ & \text { ce moment seul se focaliser Cop-Pass aida ni .... } \\ & \text { pendant ce court instant où il détourne son attention }\end{aligned}$ pendant que

En (11), le maintien de la référence s'exprime par un enchaînement parataxique avec un énoncé verbal. Le sujet syntaxique du verbe n'est pas exprimé dans l'énoncé, c'est la dernière entité saillante mentionnée qui est sujet.

Il est important de noter que l'entité saillante n'est pas nécessairement le sujet syntaxique de l'énoncé ; elle exprime une sorte de contrôle sur une situation secondaire. Par exemple en (12) ci-dessous, la fuite et la 
disparition du chat sont deux événements qui impliquent le chat et sont mentionnés comme tels dans le récit au moyen de deux énoncés constitués d'un verbe final et d'un sujet syntaxique exprimé par neko GA, ces deux énoncés sont toutefois précédés de l'entité saillante ottokonoko WA qui indique un contrôle logique de l'entité saillante < garçon> sur les deux situations dans sa portée. En ce sens, WA détache bien une entité d'un point de vue logique alors que GA intègre l'entité dans l'énoncé en la rendant sujet syntaxique.

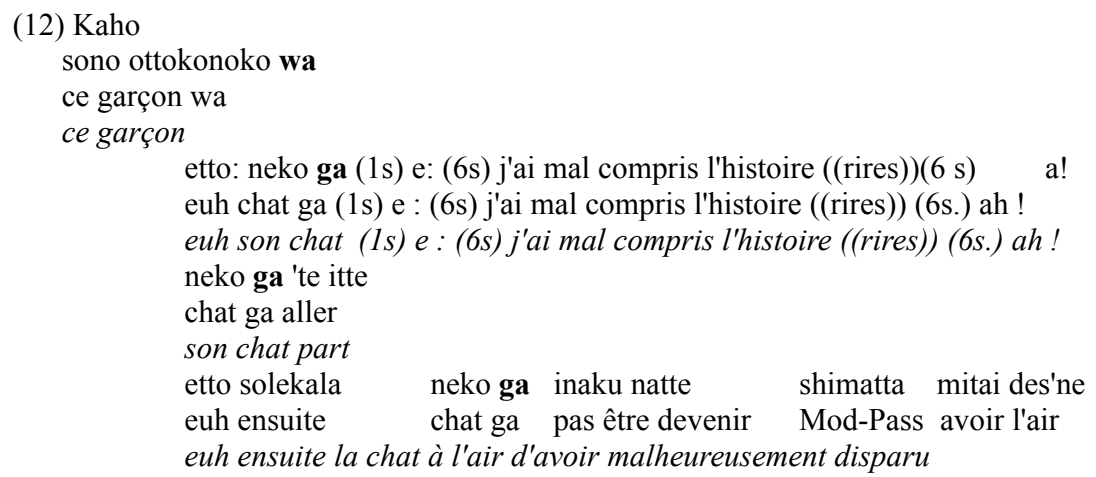

Comment s'exprime la référence à une autre entité, ou rupture référentielle ? Nous observons deux cas de figure. D'une part, comme nous l'avons noté en (12), la référence à une situation impliquant une entité autre que l'entité promue topique dans le discours s'exprime par un nom suivi de ga. Ces moyens de structuration informationnelle construisent deux niveaux narratifs, un niveau principal avec des actions principales impliquant un ou des protagonistes principaux et un niveau secondaire imbriqué dans le niveau principal avec des actions et personnages secondaires sous contrôle des principaux. Lorsque le narrateur conçoit un enchaînement d'actions impliquant uniquement le protagoniste principal, la référence à cette entité est maintenue par la construciton d'un sujet saillant SN + WA et un enchaînement d'énoncés verbaux dans sa portée. Dans la conception d'actions parallèles impliquant deux protagonistes, la construction SN + WA est suivie d'énoncés constitués d'un sujet syntaxique et d'un verbe final. Dans ce cas, le sujet syntaxique exprimé par SN + Ga peut-être le même que le sujet saillant ou différent, comme dans l'extrait (13) suivant :

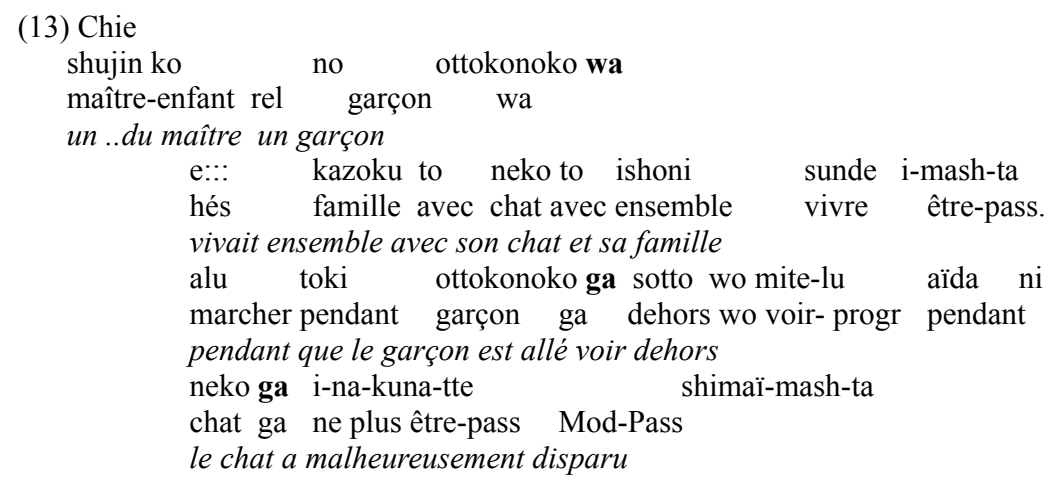

Dans cet extrait, la fuite du chat est conçue comme une action secondaire dans un cadre principal dominé par l'entité <garçon>. Un autre cas de figure est la promotion de la nouvelle référence à un statut saillant au moyen de WA. Dans le récit de Misa en (14) par exemple, la fuite du chat est exprimée dans un énoncé qui promeut $<$ chat $>$ en entité saillante :

\footnotetext{
(14) Misa

tanoku wa

gars wa
} 


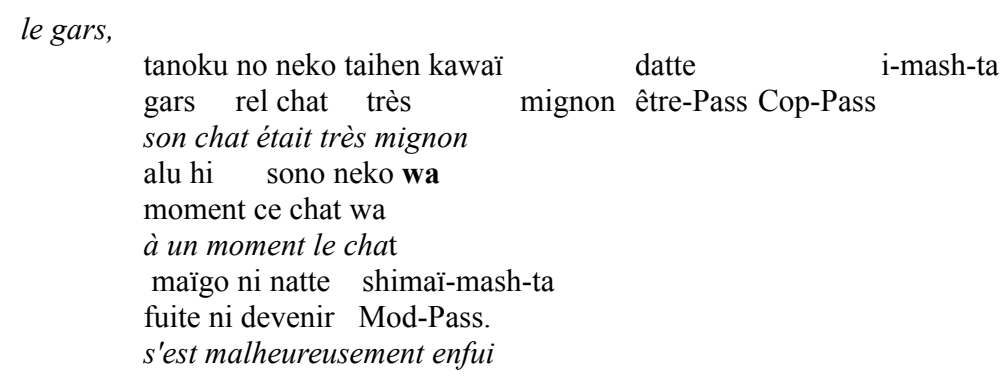

La saillance est donc bien une notion exprimée dans tous les débuts de récit en japonais au moyen d'un syntagme nominal en tête d'énoncé suivi de la particule WA. Un effet syntaxique de la construction saillante est qu'elle autorise la mention de situations impliquant l'entité au moyen de verbes situés dans sa portée, sans que l'antéposition d'un nom ou pronom ne soit nécessaire. La continuité référentielle ne nécessite donc pas l'emploi d'un élément linguistique référant au sujet du verbe de la proposition, le sujet par défaut est le dernier élément nominal précédé de $w a$. En cas de rupture référentielle ou introduction dans le récit d'un autre protagoniste en fonction d'agent, on observe l'usage d'un nom suivi de la particule $w a$, ce qui fait de l'entité auquel il réfère une entité saillante à propos de laquelle le locuteur fait des assertions, ou suivi de la particule ga qui intègre le nom à l'énoncé comme sujet du verbe sans remettre en cause l'unité saillante au moment de l'énonciation.

\section{2. L'expression de l'identifiabilité en français et en japonais}

Le récit impliquant seulement deux protagonistes, on peut penser que la seule chose essentielle à la structuration informationnelle du récit est de savoir qui du garçon et du chat fait quoi, et non de marquer linguistiquement à chaque énoncé si le protagoniste $<$ chat $>$ ou $<$ garçon $>$ est celui déjà évoqué ou un nouveau et s'il s'agit bien toujours du chat qui appartient au garçon.

\subsubsection{En français $L 1$}

En français néanmoins, l'identifiabilité des protagonistes du récit est systématiquement marquée au sein du syntagme nominal au moyen d'un déterminant. La première mention de <garçon> s'exprime systématiquement au moyen d'un SN indéfini, un ptit garçon, et les mentions nominales suivantes - qui suivent une référence à un autre protagoniste- sont marquées au moyen d'un SN défini, le ptit garçon. L'article indéfini marque le fait que l'entité mentionnée n'est pas supposée identifiable du destinataire, comme ce peut-être le cas lors de la première mention si le référent est exclu de la situation d'énonciation. L'article défini marque au contraire l'identifiabilité. Dans le cadre du récit, un référent peut être identifiable du fait qu'il a déjà été introduit, comme c'est le cas des références aux protagonistes ici analysées.

Dans le cas des références au second protagoniste, <chat $>$, l'expression de la référence et de son identifiabilité dépend aussi du lien supposé entre $<$ garçon $>$ et $<$ chat $>$. En effet, un lien d'appartenance peut être conçu entre les deux protagonistes, ce qui conduit à l'emploi d'un déterminant possessif devant le nom et ce, dès l'introduction de la référence :

\section{(15) L3}

aloreuh c'est un ptit garçon qui joue avec son cha dans sa maison euh

Dans la plupart des cas, la référence à <chat> est introduite dans la dépendance morphosyntaxique et sémantique de un ptit garçon, d'où le syntagme nominal son chat. Dans un récit toutefois, <chat $>$ est introduit comme une entité non identifiable:

(16) L4

à l'oral d'accord donque on peut voir un p'tit garçon qui euh s 'approche d'un chat 
Les occurrences suivantes de <chat>, identifiables, sont réalisées au moyen d'un syntagme possessif, son chat, ou défini, le chat, comme en (17) ci-dessous:

\section{(17) L2}

euh eum quand il se retourne de nouveau vers son chat euh le chat a disparu.

L'énoncé ci-dessus témoigne du fait que le déterminant du SN référant à <chat> varie en fonction de la fonction syntaxique du SN. L'emploi du déterminant possessif est plus fréquente lorsque le SN est en position de complément et le petit garçon en fonciton de sujet du verbe. En revanche, lorsque le SN référant à <chat $>$ est en position de sujet, l'emploi du défini est plus fréquent.

\subsubsection{En japonais $\mathrm{L} 1$}

En japonais L1, les récits ne présentent pas de moyens linguistiques systématiques pour marquer une entité supposée non identifiable de l'allocutaire et une entité supposée identifiable dans la situation énonciative de narration du fait notamment d'une mention préalable. Comme on peut l'observer dans le récit de Chie déjà mentionné en (6), la première référence à $<$ garçon $>$ et à $<$ chat $>$ se fait au moyen des noms ottokonoko et neko et la référence suivante se fait aussi au moyen des noms ottokonoko et neko. Ces noms sont liés au reste de l'énoncé au moyen de particules ( $w a, g a$ ) et prépositions ( $t o$ ) qui n'expriment pas l'identifiabilité au sens où nous l'avons définie, mais intègrent de façon hiérarchique les entités à l'énoncé.

On relève en revanche un moyen, non systématique, d'identifier un référent comme le même référent mentionné auparavant, il s'agit du déterminant démonstratif anaphorique sono dans le paragraphe suivant :
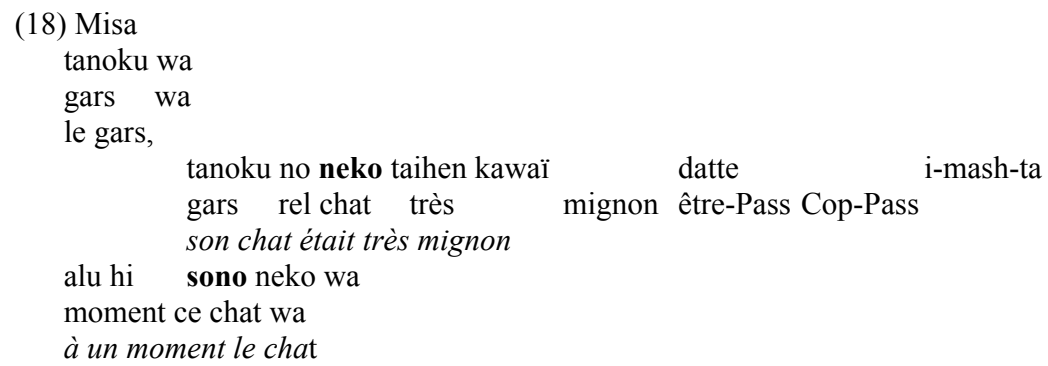

Dans cet énoncé, l'emploi de sono préposé au nom suivi de la marque de saillance wa interroge : en effet, sono est-il une marque de saillance ou d'identifiabilité ? De la même façon, même si le lien de dépendance entre <chat> et <garçon> n'est pas marqué de façon systématique, il est inductible syntaxiquement par l'inscription de l'énoncé impliquant l'entité chat dans la portée de l'entité saillante $<$ garçon> comme en (16). Dans ce premier énoncé, le syntagme verbal <être très mignon> concerne $<$ chat $>$; par conséquent le syntagme initial nominal tanoku wa véhicule, avec le complément du nom, tanoku no neko, un lien de dépendance entre <garçon> et <chat>. Encore une fois, les moyens qui expriment la saillance suggèrent aussi des liens sémantiques entre les entités.

\subsection{L'expression de la saillance et de l'identifiabilité en français $L 2$}

De façon attendue, les débuts de récit en français L2 sont plus courts qu'en français L1. Ils constituent néanmoins un matériau suffisant pour observer les principes qui guident les productions.

\subsubsection{L'expression de la saillance}

Comme nous l'avons montré en 5.2.1., les indices d'expression de la saillance activés dans le début du récit du chat perdu sont, en français, la construction relative présentative et l'emploi d'un pronom personnel sujet pour référer à une entité exprimée par un nom sujet de la proposition précédente. Nous 
allons examiner si les locuteurs de langue première japonais ont recours à ces moyens discursifs et morphosyntaxiques d'expression de la saillance en français L2.

L'analyse du premier ensemble de récits à t1 montre que la première entité mentionnée est $<$ garçon $>$ dans deux types de construction, SN- Verbe - SN/SPrép comme en (19) dans 8 cas sur 9 et Verbe de copule SN comme en (20) dans un cas.

(19) Kao

etto le garçon jou/e/ avec chat

(20) $\mathrm{Ai}$

e : garçon il y a une garçon ah un garçon et une che une chat un chat une chatt le chat

Nous ne relevons aucune construction présentative relative dans les récits et on pourrait être tenté de conclure que les apprenants ne maîtrisent pas le principe de saillance.

L'analyse des moyens de référer en position sujet à une entité qui est déjà exprimée dans l'énoncé précédent dans un syntagme nominal en position sujet montre une tendance nette à employer un pronom personnel sujet comme en (21):

(21) Rei 1

un garçon joue jou/e/ avec un chat

/e/ ::: ileu il vu il vu il veut euh +++ ah il ne veut PAS un chat

Dans le cas de figure où le locuteur fait référence, en position sujet de l'énoncé à une entité qui est mentionnée dans l'énoncé précédent dans une position syntaxique autre celle du sujet, les locuteurs nippophones emploient majoritairement, nom pas un pronom, mais un syntagme nominal, comme Yuk en (22) :

\section{(22) Yuk 1}

le garçon aime le chat

MAIS le chat est allé

Parmi les 6 cas relevés de référence en fonction sujet à une entité déjà sujet de l'énoncé précédent, le pronom est employé dans 5 cas, un syntagme nominal dans l'autre cas. Parmi les 8 cas de référence en fonction sujet à une entité ayant une fonction autre que sujet dans l'énoncé précédent, un syntagme nominal est employé dans 7 cas, un pronom dans le cas restant. Sachant qu'aucun transfert grammatical n'est concevable entre le japonais et le français, ces occurrences témoignent de l'accessibilité du principe de saillance discursive pour les locuteurs qui utilisent ces constructions.

L'analyse des récits à $t 2$, recueillis deux mois plus tard après un séjour immersif en France, confirme et renforce ces conclusions. Dans les premiers énoncés des récits, la construction Cop -SN est plus fréquente ( 3 occurrences sur 9 récits) si bien que l'on peut se demander en (23) si Kak n'emploie pas une proto-CRP.

\section{(23) Kak}

il a le chat il a une chatt cha est parti

Quant aux pronoms, ils sont employés pour référer à une entité saillante encodée en fonction sujet dans l'énoncé précédent dans 7 cas sur 9 , ce qui confirme un usage qui tient compte de l'échelle de saillance construite par la distribution syntaxique des noms dans l'énoncé.

L'absence de construction présentative à t1 ne peut donc constituer un indice suffisant pour vérifier si les apprenants nippophones expriment dans le discours ce principe clé de construction de la structure informationnelle qu'est le principe de saillance discursive. En effet, l'analyse de l'usage des pronoms personnels sujets montre donc, non seulement l'usage systématique en contexte obligatoire de ces unités morphosyntaxiques absentes du japonais mais aussi un usage approprié d'éléments complexes. Cette complexité a été mise en évidence en 5.2.1. par le fait qu'à deux reprises, nous avons observé que les locuteurs natifs dans leur production spontanée en français L1 s'autocorrigent, emploient d'abord un pronom puis un nom, pour lever l'ambiguité référentielle du pronom. L'observation de constructions proto-présentatives à $\mathrm{t} 2$ suggère ainsi qu'en termes de moyens linguistiques, l'expression pronominale de la saillance est première par rapport à l'expression discursive (CRP). 


\subsubsection{L'expression de l'identifiabilité}

Le principe d'identifiabilité énonciative est, dans la construction de la structure informationnelle en français, un deuxième principe clé exprimé au moyen d'une distribution prénominale des articles définis et indéfinis qui n'a pas d'équivalent en japonais. Un contexte d'observation privilégié de l'expression de l'identifiabilité de la référence nominale est donc le cotexte de référence à une entité introduite ou réintroduite dans le discours au moyen d'un nom.

En français L1, les 6 syntagmes nominaux mentionnant <garçon> pour la première fois sont, dans les 6 récits analysés, tous indéfinis : un ptit garçon tandis que les 12 syntagmes nominaux employés dans le contexte de réintroduction de cette entité sont tous définis: le petit garçon. Sachant cette claire distribution des articles, nous pouvons considérer ces deux contextes comme des contextes diagnostics suffisants pour analyser l'expression de l'identifiabilité en français L2.

Dans les 9 récits analysés en français L2, les articles, qui sont des catégories absentes du japonais, sont employés mais leur distribution se distingue de celle observée en L1. Lors du premier enregistrement à t1, 5 syntagmes nominaux sont indéfinis (un garçon) contre 3 définis. Lors du deuxième enregistrement, deux mois plus tard, l'article indéfini est employé par 3 narrateurs seulement, les autres ayant recours à l'article indéfini ou à un autre moyen (abence de déterminant ou pronom). La variation des données montrent que l'introduction d'une entité n'est globalement pas associée à l'emploi d'un article indéfini.

Tableau 1. Référence à <garçon> selon l'identifiabilité énonciative

\begin{tabular}{|l|l|l|l|l|l|l|}
\hline & \multicolumn{2}{l|}{1 1̀̀re mention } & \multicolumn{2}{l|}{ Mentions suivantes } \\
& UN & LE & autre & LE & UN & autre \\
\hline FL1 & $6 / 6$ & & & $12 / 12$ & & \\
\hline \hline FL2, à t1 & $5 / 9$ & $3 / 9$ & $1 / 9$ & & $1 / 1$ & \\
\hline FL2, à t2 & $3 / 9$ & $3 / 9$ & $3 / 9$ & $3 / 4$ & & $1 / 4$ \\
\hline
\end{tabular}

Le principe selon lequel l'article défini exprime en français la référence à une entité identifiable, par exemple une entité réintroduite, n'est pas vérifiable à t1 sachant la quasi abence de cotexte discursif de réintroduction. A t 2 toutefois, nous observons qu'en cotexte de réintroduction, l'article défini est employé dans 3 cas sur 4. Sachant que l'article défini est aussi utilisé dans un tiers des contextes de première mention analysés, on ne peut conclure, globalement, à une distribution appropriée des articles définis et indéfinis.

On peut également souligner l'émergence du déterminant possessif devant le nom chat dans les récits recueillis à $\mathrm{t} 2$. Deux apprenantes qui n'emploient pas le possessif devant l'entité <chat $>$ lors du premier enregistrement l'emploient au deuxième, à l'instar de Kazumi en (24 a et b) :

(24a) Kazumi 1

un garçon a joué avec un chat

pendant il a regardé dehors un chat est parti

et il est desperise parc'que un chat est disparu

(24b) Kazumi 2

b. un garçon a joué avec son chat

comme il étè letené son cham népala

L'emploi du déterminant possessif pour identifier la référence en lien avec l'autre entité <garçon> mentionnée est d'emblée approprié. Sachant que le déterminant possessif n'est pas une catégorie syntaxique existante en japonais, au même titre que les articles définis et indéfinis, ce phénomène montre 
d'abord que ce fait n'est pas un obstacle à son acquisition en français L2. L'émergence de ce déterminant dans les récits recueillis après un séjour immersif en France suggère également un effet de l'exposition au français sur l'acquisition de ce moyen de structuration informationnelle. Enfin, l'emploi approprié du déterminant possessif montre également que pour des apprenants nippophones, le déterminant possessif est plus apprenable que les articles définis et indéfinis. Il est tentant de rapprocher ce phénomène de celui de l'usage des pronoms. En effet, même si le déterminant possessif est une catégorie absente du japonais, il exite dans les récits analysés en japonais L1 des moyens explicites d'exprimer la relation de dépendance entre deux entités, <garçon> et <chat> en l'occurence, comme le complément du nom (tanoku no neko, gars de chat) ou le placement du nom neko (chat) dans la portée immédiate du nom ottokonoko (garçon) rendue saillante par la particule wa (sono ottokonoko wa neko ga itte... ce garçon, chat il y a ....). L'usage du possessif à t2 est donc un phénomène qui suggère la prééminence des principes sémantiques de haut niveau dans le processus d'acquisition du français L2 par des apprenants nippophones.

Cette deuxième partie de l'analyse des récits en français L2 montre que les apprenants nippophones n'ont manifestement pas accès au principe d'identifiabilité en français. Alors que la saillance des entités est exprimée en discours à travers les pronoms, l'identifiabilité des référents n'est pas exprimée au moyen des articles définis et indéfinis. Au terme de l'analyse, la distribution des articles apparaît comme une dsitribution encore largement aléatoire.

\section{Conclusion}

Le principe de saillance et le principe d'identifiabilité s'expriment en français par des constructions discursives et des indices morphosyntaxiques qui n'ont pas d'équivalents formels en japonais. Les analyses des récits en français et japonais L1 confirment cette idée. L'analyse des données de français L2 montrent toutefois que ces considérations préliminaires sont insuffisantes pour expliquer le développement de l'expression de la référence aux entités puisqu'en français L2, les apprenants nippophones n'expriment pas la propriété identifiable du nom mais expriment en revanche sa propriété saillante comme en témoigne l'usage adéquat des pronoms personnels sujets dans le discours narratif des apprenants nippophones. Si les mêmes causes doivent produire les mêmes effets, il s'ensuit que l'acquisition de l'expression de la saillance et l'acquisition de l'expression de l'identifiabilité en français L2 sont, pour des apprenants nippophones, deux processus distincts. L'origine de cette distinction ne pouvant pas résider dans les propriétés morphosyntaxiques de la langue source (absence d'articles ou de pronoms), l'hypothèse morphosyntaxique selon laquelle l'absence d'une catégorie morphosyntaxique dans une langue source entraîne des difficultés d'usage en langue cible est partiellement rejetée. L'analyse de la référence aux entités dans les débuts de récit montre en revanche que l'hypothèse sémantique selon laquelle le processus acquisitionnel est guidé par des catégories sémantiques est validée. Les expressions de la référence aux entités dans le français L2 des apprenants nippophones s'explique par le fait que certains principes sémantiques de haut niveau comme le principe de saillance ou celui de dépendance entre deux unités est exprimé en japonais, même si c'est par des moyens syntaxiques et discursifs non comparables avec les moyens linguistiques employés en français.

Cette analyse montre qu'il y a lieu de lever les ambiguités liées à la notion de topique en distinguant la saillance discursive et d'identifiabilité énonciative et affirme donc la prééminence des catégorie sémantiques dans le processus d'acquisition de l'expression de la référence aux entités. Les récits recueillis après une exposition intensive au français parlé témoignent également de l'influence respective des langues source et cible dans le processus acquisitionnel. La possibilité d'exprimer la saillance en japonais constitue un facteur favorable à l'appropriation des règles d'usage des pronoms, marqueurs de saillance, en français. Le fait que le japonais dispose de moyens linguistiques de rendre une unité référentielle saillante dispose à acquérir l'expression pronominale de la saillance en français. L'emploi par quelques apprenants d'un énoncé introductif présentatif suggère aussi l'apprenabilité à ce stade de la construction présentative. La distribution fluctuante des articles ne remet pas en cause le fait que le principe de prénominalisation est acquis. En effet, dans les débuts de récits analysés, la plupart des apprenants emploient dans la plupart des occurences des prénoms ou déterminants devant le nom. 
L'existence de quelques prénoms en japonais a pu contribuer à l'acquisition de ce principe. En revanche, le principe de distribution des articles selon lequel une entité supposée non identifiable de l'allocutaire est introduite au moyen d'un article indéfini et une entité supposée identifiable au moyen d'un défini, n'est pas acquise. Par exemple, la distribution des prénoms ne rend pas compte d'un marquage distinct, dans les récits, des premières mentions - supposées non indentifiables- d'une entité et des réintroductions de ces mêmes entités - par définition devenues identifiables. Ce résultat peut être mis en relation avec le marquage de la possession au moyen du déterminant possessif par deux apprenantes lors du 2ème enregistrement. Il apparaît nettement à l'issue de ces analyses que le japonais langue source n'est pas seulement un obstacle à l'acquisition de la référence aux entités en français langue seconde, comme le suggère une approche grammaticale de la question des articles. Il est nécessaire d'aborder les phénomènes d'acquisition de la morphologie nominale autrement que comme un transfert négatif d'une langue sans article vers une langue avec articles. Le japonais langue source est aussi une langue ressource qui favorise l'acquisition de l'expression de la structure informationnelle en langue cible.

Dans cette perspective, on peut considérer avec Klein (2008 : 305) que les difficultés d'acquisition du français L2 proviennent précisément de la langue cible qui encode l'identifiabilité du référent de façon redondante pour ne pas dire pathologique, comme le suggère fortement l'extrait suivant :

\begin{abstract}
« we need information structure, or rather the « aboutness distinction » of information structure. The other main dimension of information structure is along the distinction between "given information" and «new information». Here we are faced with the somewhat puzzling question why it should be important to signalize this distinction. After all, the interlocutor is not stupid and should therefore know anyway which information is new and which information is not.It is surely true that, if some referent is given in context, less descriptive information is needed in order to identify it. Therefore we may say he rather than my late uncle Richard's best friend in school, when this person was talked about before. But there is little reason to assume that « givenness » or «newness » must be MARKED, unless the speaker has reason to assume that the adressee suffers from Alzheimer. »
\end{abstract}

\title{
Références bibliographiques
}

Chafe W. (1976) Givenness, contrastiveness, definiteness, subjects, topics and point of view. In Charles Li (ed.): Subjects and Topics. New York: Academic Press.

Epstein R. (2002). The definite article, accessability and the construction of discourse referents. Cognitive Linguistics 12-4, 333-378

Hendricks H. (2000). The Acquisition of Topic Marking in L1 Chinese and French L1 and L2. Studies in Second Language Acquisition 22, 367-389.

Hidetake I. (2010). Le défini et l'indéfini dans une langue sans article. Langue française 171, 13-26.

Jarvis S. (2002). Topic continuity in L2 English article use. Studies in Second Language Acquisition 24, $387-418$.

Klein W. (2008). The topic situation. In B. Ahrenholz, U. Bredel, W. Klein, M. Rost-Roth, \& R. Skiba (eds.), Empirische Forschung und Theoriebildung: Beiträge aus Soziolinguistik, Gesprochene-Sprache- und Zweitspracherwerbsforschung: Festschrift für Norbert Dittmar, Frankfurt am Main, Lang, 287-305.

Lebas-Fraczak L. (2009). Description «communicative» des déterminants français en vue de la didactisation. Les Cahiers de l'Acedle 6 : 2, 69-100.

Lenart E., Perdue C. (2004). L'approche fonctionnaliste : structure interne et mise en ouvre du syntagme nominal, AILE 21, 85-121.

Lenart E. (2012). The Grammaticalisation of Nominals in French L1 and L2 : A Comparative Study of Child and Adult Acquisition. In Watorek, M., Benazzo, S. et Hickmann, M. (éds). Comparative Perspectives to Language Acquisition: Tribute to Clive Perdue. Multilingual Matters, 420-440. 
Klingler D. (2003). Spécificité du dispositif créé par le marquer WA en japonais et comparaison avec le français. Travaux de linguistique, 163-179.

Lambrecht K. (1994). Information Structure and Sentence Form. Cambridge : Cambridge University Press.

Lambrecht K. (2000). Prédication seconde et structure informationnelle: la relative de perception comme construction présentative. Langue française 127, 49-66.

Sleeman P. (2004). The acquisition of definiteness distinctions. Linguistics in the Netherlands 21, 158-168.

Tamba I. et Terada A. (1991). La phrase japonaise et son double dispositif d'intégration des noms: les particules dites relationnelles et casuelles», Langages 104, 33-45.

Trévisiol P. (2003). Problèmes de référence dans la construction du discours par des apprenants japonais du français, L3. Thèse de Doctorat, Université Paris VIII. 
${ }^{1}$ Ces données ont été recueillies et transcrites dans le cadre d'une coopération franco-japonaise, avec l'aide de Fumiya Ishikawa (Université de Yokohama), Dominique Klingler (Université de Paris III), Ako Nishimura (Université de Shirayuri) et de tou-te-s les étudiant-e-s qui, au Japon et en France, ont accepté de façon désintéressée les activités langagières que nous leur proposions. 